The Communications Web site, http://cacm.acm.org, features more than a dozen bloggers in the BLOG@CACM community. In each issue of Communications, we'll publish selected posts or excerpts.

\title{
'Generation CS' Drives Growth in Enrollments
}

\author{
Undergraduates who understand the importance of computer science \\ have been expanding the CS student cohort for more than a decade.
}

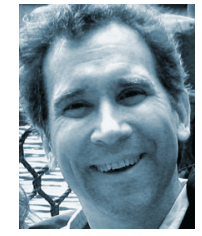

Generation CS: When Undergraduates Realized They Needed Computing By Mark Guzdial http://bit.ly/2qiMahP

March 28, 2017

The new Computing Research Association (CRA) report "Generation CS: Computer Science Undergraduate Enrollments Surge Since 2006" (http:// cra.org/data/Generation-CS/) describes the dramatic increase in enrollments in computer science (CS) over the last 11 years, with an especially rapid increase since 2009. Sixty percent of academic units surveyed more than doubled their enrollment in that time. The report describes a new generation of undergraduate students who realize the importance of computing education.

The CRA committee that assembled the report carefully analyzed the data in terms of size of the department (for example, number of tenure-track faculty), type of department (for example, Ph.D.-granting or not), and where it characterizes growth in terms of majors vs. non-majors. The bottom line is reflected in this quote:
The current surge of CS majors is pervasive. Large and small academic units, in public and private institutions, have been affected similarly. Doctoral granting and non-doctoral granting units are affected, though doctoral granting units to date have seen larger increases. While academic units are taking a range of actions to handle the increased enrollment, percentage increases in tenure-track faculty are about $1 / 10^{\text {th }}$ of the increase in the number of majors.

I found several surprises in the report:

- Non-major enrollment is also increasing, and at all levels. One might expect the number of non-CS majors to increase at the intro level, but there are also huge increases at the mid and upper levels of the undergraduate curriculum. For units that track the data, the growth in CS minors is similarly dramatic.

- Efforts to diversify computing are failing in the face of the enrollment increase. A recent report from Code.org ("University computer science finally surpasses its 2003 peak!," https://medium.com/anybody-can-learn/universitycomputer-science-finally-surpasses-its2003-peak-ecefa4c8d77d) shows that the number of CS graduates has finally sur- passed the number from 2003, the peak of CS graduate production. Unfortunately, the number of female CS graduates is even less than in 2003. The evidence in "Generation CS" suggests that there are women in the introductory classes, but we are not retaining them into the mid and upper levels of the undergraduate curriculum. The evidence suggests the percentage of Black/African-American students in CS is declining, while Hispanic/Latino percentage share is increasing. A positive sign is that the departments that report taking actions to increase diversity are more diverse.

- Departments are having to tighten their belts in response to the increase in enrollment. Schools are not really helping yet. As you can see in the accompanying table, faculty increases are nowhere near the enrollment increases. CRA is offering to share the data from the report with any department that would like to use this data to argue for resources. Departments are canceling low-enrollment classes, increasing class sizes, using more adjunct faculty, using more undergraduate students as teaching assistants, and using more graduate students as instructors. 
Google has funded several efforts to respond to the enrollment increases without sacrificing diversity gains. Chris Stephenson has a blog post describing these efforts (https://research. googleblog.com/2017/02/the-cs-capacityprogram-new-tools-and.html), with links to more information. Until we can convince schools to increase resources to departments, developing strategies like these and sharing them are our best chances to manage "Generation CS" without losing ground on our efforts to provide CS education to all students.

\section{Comments}

If the number of female CS graduates is decreasing, or the number of male CS graduates is decreasing, you cannot assume that the only reason is that universities are not doing enough to recruit them, or encourage them, into the field.

There are many possible reasons outside of the purview of computing, and beyond the competence of the science and practice of computing. Not all of them are even addressable by policies in academia.

There is, however, one factor that is not even mentioned here. There are computing "boot camps" popping up all over the place in the U.S. and outside the U.S. These students are not being counted I'll bet because this article only talks about majors and minors and "diversity" numbers. I personally know several women who have been through these. And the cost is a LOT less in both money and time-to-jobs.

In other words, computing as a practical skill is gaining ground, and private-sector computing (and even many government entities) are interested more in proper results than in credentials.

Academia has had it pretty good with all the federally guaranteed loans pouring into its treasuries after World War II, but the burden has been put upon the over-taxed middle class and even worse on college graduates. Who can blame the victims for seeking alternatives?

The decline after the peak in 2003 mentioned here, we all know why that happened in the U.S. at least. I'll bet it was different in India. Starting with the Y2K projects just before the century digit turned over, the rush to finish and the new Internet infrastructure began the use of remote resources. And there began a decline that continued with some of my coding colleagues training H1B's to replace them. (Illegally but

Cumulative percent growth of CS majors and instructional faculty since 2006.

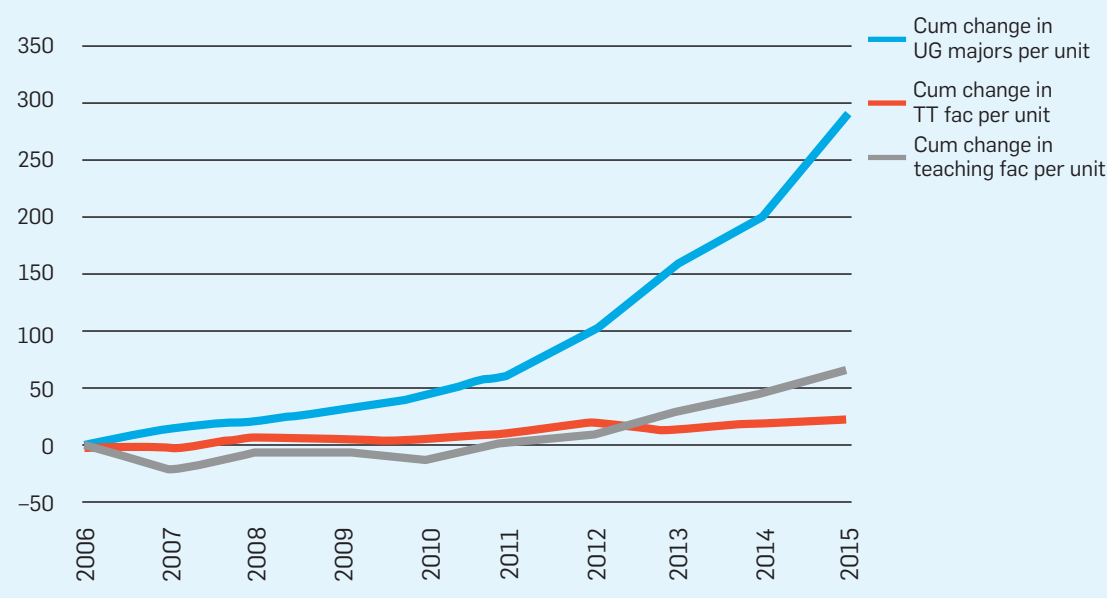

Source: CRA Taulbee Survey

never prosecuted at the time). Philosophically I am a culture-aware libertarian, but hate it when the public is lied to.

It may be that smaller companies that do not have resources to get computing help from overseas are expanding the market for computing skills. It doesn't matter. Getting government out of the picture altogether would remove the warp.

The freak-out about diversity numbers suggests that young girls already know they have no barrier to computing fields and are opting for other studies. I have three daughters who fit this description, and I hate this narrative that stereotypes my daughters based on the warped thinking that something is wrong if females don't make the same choices as males. That is biological nonsense, and studies have shown the differences are innate even from the womb. If it is clear that there are no barriers and that females even get the advantage of extra attention for being a minority in the field, why not take the win and run with it instead of seeking blowback against all the pressure?

- Cassidy Alan

I don't assume that universities are not doing enough to recruit, encourage, or keep women in computing. I know that because there is a large body of computing education research showing that it's true.

I encourage you to look at the excellent books about the work at Carnegie Mellon University where they successfully have recruited women so that over $40 \%$ of their CS class is female. Or, check out the articles on Harvey Mudd College where they are over $50 \%$ female. There is a project called BRAID (https://www.hmc.edu/abouthmc/2014/09/24/harvey-mudd-launchesinitiative-increase-diversity-computerscience/) to teach other CS departments what Harvey Mudd figured out. It's within the CS departments' control to improve their gender diversity.

The research on "boot camps" is devastating. Many "boot camp" students take repeated boot camps because they don't learn enough CS and can't get jobs.

The reason why few women pursue computing in the U.S. has nothing to do with biology. At Qatar University, computer science is $75 \%$ female and computer engineering is 100\% female (https:// computinged.wordpress.com/2010/10/18/ latest-enrollment-numbers-at-qataruniversity-big-gender-imbalance/). The gender balance in CS is much more about culture than it is about biology.

I have two daughters myself. Both have tried computer science classes and been quite successful in them. Neither are choosing to get CS degrees. There is nothing wrong with them for pursuing other subjects. As a computing education researcher who studies broadening participation issues, I can list for you all the things that their CS departments did wrong-not recruiting, not encouraging, not keeping women. The problem is with the CS departments, and the data and research studies back me up.

$$
\text { -Mark Guzdial }
$$

